\title{
Altered DNA methylation is associated with aberrant stemness gene expression in early-stage HNSCC
}

\author{
TAKATSUGU SUZUKI $^{1}$, HIROSHI YAMAZAKI ${ }^{1}$, KAZUFUMI HONDA $^{2}$, EIJITSU RYO $^{3}$, \\ AKIHIRO KANEKO $^{1}$, YOSHIHIDE OTA ${ }^{1}$ and TAISUKE MORI ${ }^{3,4}$ \\ ${ }^{1}$ Department of Oral Surgery, Tokai University School of Medicine, Isehara, Kanagawa 259-1193; \\ Divisions of ${ }^{2}$ Biomarker for Cancer Early Detection and ${ }^{3}$ Molecular Pathology, National Cancer Center Research Institute; \\ ${ }^{4}$ Department of Pathology and Clinical Laboratories, National Cancer Center Hospital, Tokyo 104-0045, Japan
}

Received February 12, 2019; Accepted July 17, 2019

DOI: $10.3892 /$ ijo.2019.4857

\begin{abstract}
Head and neck squamous cell carcinoma (HNSCC) is characterized by morphological and functional cellular heterogeneity, which are properties of progenitor cells, as opposed to cell alterations caused by accidental expression of stem cell-related molecules. The expression levels of stemness molecules and their distribution in HNSCC are unclear. As regards sporadic cellular heterogeneity, methylation is an important factor for transcriptional regulation in tumors. Integrative screening analysis of mRNA expression and altered methylation status was performed with original microarrays in 12 tumor and non-tumor pairs of oral squamous cell carcinoma (SCC) cases. From this data set, genes regulated via aberrant DNA methylation and classified proteins were validated by function clustering. Olfactomedin 4 (OLFM4), known as an intestinal stemness molecule and cell-cell adhesion factor, was found to be highly expressed in tumors, with an mRNA expression ratio [tumor/normal $(\mathrm{T} / \mathrm{N})$ ] of 40.7686 and low methylation $(-18.02 \%)$ in the promoter region. In addition, the OLFM4 expression levels increased following treatment with the demethylating agent 5-azacytidine in two HNSCC cell lines. Furthermore, the expression levels of OLFM4 in 59 cases of early-stage tongue SCC were analyzed using immunohistochemistry to examine protein expression corresponding to the histopathological definition of tumors and to evaluate prognosis. The aberrant stemness gene expression caused by altered DNA methylation appeared to regulate early-stage HNSCC characteristics. The results of the present study indicated a correlation between OLFM4 expression and promoter methylation, and suggest that it plays an important role in tumor cell heterogeneity in HNSCC.
\end{abstract}

Correspondence to: Dr Taisuke Mori, Department of Pathology and Clinical Laboratories, National Cancer Center Hospital, 5-1-1 Tsukiji, Chuou, Tokyo 104-0045, Japan

E-mail: tamori@ncc.go.jp

Key words: head and neck squamous cell carcinoma, gene expression, stemness gene, olfactomedin 4

\section{Introduction}

Head and neck squamous cell carcinoma (HNSCC) is the sixth most common type of cancer and affects an estimated 600,000 individuals worldwide annually (1). Due to its anatomical location, HNSCC may cause problems postoperatively, such as difficulty in speaking, swallowing and eating. Patients with advanced HNSCC require aggressive treatment, as HNSCCs are often associated with extensive local invasion and frequent regional lymph node metastasis (2). Therefore, early detection, in-depth understanding of the characteristics of cancer cells and accurate diagnosis are crucial for successful treatment. In addition, comprehensive analysis of HNSCC may enable the development of novel diagnostic aids, such as molecular biomarkers, and provide novel therapeutic targets.

The human OLFM4 gene was first cloned from human myeloid progenitor cells (3), and it is highly expressed in intestinal stem cells. The expression of OLFM4 is upregulated in gastric $(4,5)$ and pancreatic $(6)$ cancer, whereas it is downregulated in prostate cancer (7), colon cancer and leukemia (8). The OLFM4 gene encodes the secreted protein OLFM4, which is normally expressed in the prostate gland, bone marrow, small intestine, colon and pancreas $(3,9)$. OLFM4 expression is associated with the differentiation and progression of gastric cancer $(10,11)$ and colon adenocarcinoma (12). A previous study reported that the induction of OLFM4 expression in cancer cells exerts an anti-apoptotic effect and promotes proliferation of cancer cells (13). In addition, OLFM4 promotes $\mathrm{S}$ phase transition and proliferation of cancer cells and regulates tumor cell adhesion and migration (6). Moreover, OLFM4 has been considered as a novel biomarker for gastrointestinal cancers $(4,11,14)$.

Gene expression is regulated by genetic and epigenetic mechanisms. The effects of epigenetic modifications on aging, growth and development, and certain diseases, have already been reported. Epigenetic change is a type of gene abnormality that does not involve a change in gene sequence, but rather histone modification and DNA methylation.

Previous studies have demonstrated that DNA hypomethylation of specific genes is associated with several types of cancer, including HNSCC (15-18). In addition, promoter hypermethylation of tumor suppressor genes, such as p16, has 
been observed during the early stages of HNSCC carcinogenesis (19-22). However, only few studies to date have investigated the interconnection between epigenetics and HNSCC.

The present study focused on gene expression regulation via aberrant DNA methylation in HNSCC, aiming to elucidate the effect of DNA methylation on HNSCC carcinogenesis by comprehensive analysis of gene expression and DNA methylation using microarray and methylation microarray. In particular, OLFM4, a known stemness gene, was selected to elucidate the effect of DNA methylation on stemness gene expression in cancer stem cells (CSCs) during carcinogenesis. The correlation between OLFM4 expression and the clinical characteristics of HNSCC is discussed below.

\section{Materials and methods}

Tissue samples. Formalin-fixed paraffin-embedded (FFPE) specimens were collected from 59 cases of tongue SCC (40 well-differentiated, 7 moderately differentiated and 12 poorly differentiated SCCs). All cases were treated by resection, without chemotherapy or radiotherapy. These 59 cases, in which the tumor size was $<4 \mathrm{~cm}$ and there was no metastasis (T1, and T2, N0, M0), were used for overall survival evaluation. The tumor tissues were surgically resected between April 1998 and March 2006 at the National Cancer Center Hospital (Tokyo, Japan). Informed consent was obtained from the 59 adult patients ( 36 male and 23 female patients, mean age 60.1 years, range 28-84 years) and the Ethics Committee of the National Cancer Center Hospital approved the study protocol (approval no. 2010-077). The present study is retrospective and included the use of previously stored tissue samples.

Tissue microarray (TMA) for immunohistochemistry (IHC) analysis. Formalin-fixed paraffin-embedded specimens from the 59 cases were collected and analyzed.

The TMA blocks were cut into $4-\mu \mathrm{m}$ sections. The deparaffinized sections were subjected to hematoxylin and eosin (HE) and IHC staining. IHC was performed with anti-OLFM4 primary antibody (1:1,000; LS-B2055; Life Span Bio Sciences, Inc.). Each section was exposed to $0.3 \%$ hydrogen peroxide for 15 min to block endogenous peroxidase activity. An automated stainer (Dako) was used for staining according to the manufacturer's protocol. The ChemMate EnVision method (Dako) was used for detection. Appropriate positive and negative controls were used. The slides were observed under a microscope (BX53, Olympus Corporation; magnification, x200 and $\mathrm{x} 400$ ) and evaluated with the modified OLFM4 staining score. The percentage of immunopositive cells was divided into three scores as follows: $<30 \%$ (score 0, negative); 30-69\% (score 1 , positive); and $>70 \%$ (score 2 , diffusely positive) (23).

RNA in situ hybridization (ISH). ISH for OLFM4 was also performed using RNAscope FFPE assay kit (Advanced Cell Diagnostics, Inc.) as described previously (24). In brief, 4- $\mu \mathrm{m}$ FFPE tissue sections were pretreated with heat and subjected to protease digestion followed by hybridization with OLFM4 probe (Hs-OLFM4, 311041). Subsequently, an HRP-based signal amplification system was hybridized to the bound OLFM4 probe and color was developed with 3,3'-diaminobenzidine tetrahydrochloride (DAB). The housekeeping gene ubiquitin $\mathrm{C}$ (UBC) and the bacterial gene DapB served as positive and negative controls, respectively. Samples with UBC signals discernible with a x10 objective lens were considered to be adequate. The present study analyzed FFPE specimens from the 59 cases collected as described above. The methylation status in clinical samples used for ISH and IHC was not analyzed.

Tissue samples for oligonucleotide microarray and methylation microarray. HNSCCs and corresponding non-cancerous squamous epithelium samples were obtained from 12 consecutive patients who underwent surgical resection at Tokai University Hospital between April 2006 and March 2008. The patients' age at onset ranged between 34 and 91 years (mean, 68.5; 6 male and 6 female patients). Tissue samples sized $\sim 5 \times 5 \mathrm{~mm}$ were collected from the tumor and non-cancerous part of the surgical specimen prior to formalin fixation. The samples were immediately stored in RNAlater (Thermo Fisher Scientific, Inc.) and stored at $-20^{\circ} \mathrm{C}$ until processed. Histological diagnosis was made according to the WHO criteria (25). Only primary cases were included, whereas patients who had received radiation or chemotherapy for HNSCC were excluded. Informed consent was obtained from all adult patients. The present study included retrospective use of previously stored tissue samples, and the Ethics Committee of Tokai University School of Medicine approved all the procedures (approval no. 16 R-183).

Oligonucleotide microarray analysis for clinical samples. Total RNA was extracted from the 12 clinical sample pairs using RNeasy Mini Kit (Qiagen). All labeled samples were hybridized to the Agilent 60-mer oligo microarray with an $8 \times 15,000$ probe format (the probe was designed by the Agilent Technologies eArray website: http://earray.chem. agilent.com/eArray/; design ID: 021445). A Gene Expression Hybridization Kit and Gene Expression Wash Buffer Kit solutions (both from Agilent Technologies, Inc.) were used for the hybridization and washing steps, respectively. The housekeeping genes GAPDH, $\beta$-actin and ISGF-3 (STAT1) of 100 probe sets were used as a normalization control set. Fluorescence intensity was calculated using Feature Extraction software, version 9.5 (Agilent Technologies, Inc.) and the data were analyzed with GeneSpring GX software, version 11.0 (Agilent Technologies, Inc.). mRNA expression ratios between tumor and normal tissue were calculated as tumor/normal $(\mathrm{T} / \mathrm{N})$ mRNA expression ratio in each gene.

Methylation microarray for clinical samples. The changes in DNA methylation in 12 clinical sample pairs were assessed using the Illumina Infinium assay with the HumanMethylation450K DNA Analysis BeadChip (Illumina, Inc.). DNA methylation levels at individual $27578 \mathrm{CpG}$ sites represented on the Illumina BeadChip were determined by measuring the fraction of methylated signal over the total signal (unmethylated + methylated fractions) in each genomic DNA sample. The OLFM4 probe is set at a CpG region located near the ATG start site.

To compare the DNA methylation levels of $\mathrm{CpG}$ sites between tumors and controls, $\mathrm{CpG}$ sites with a mean methylation difference $(\Delta \beta)$ of $>10 \%$ were considered as differentially methylated. DNA was extracted and purified from OCT-embedded 


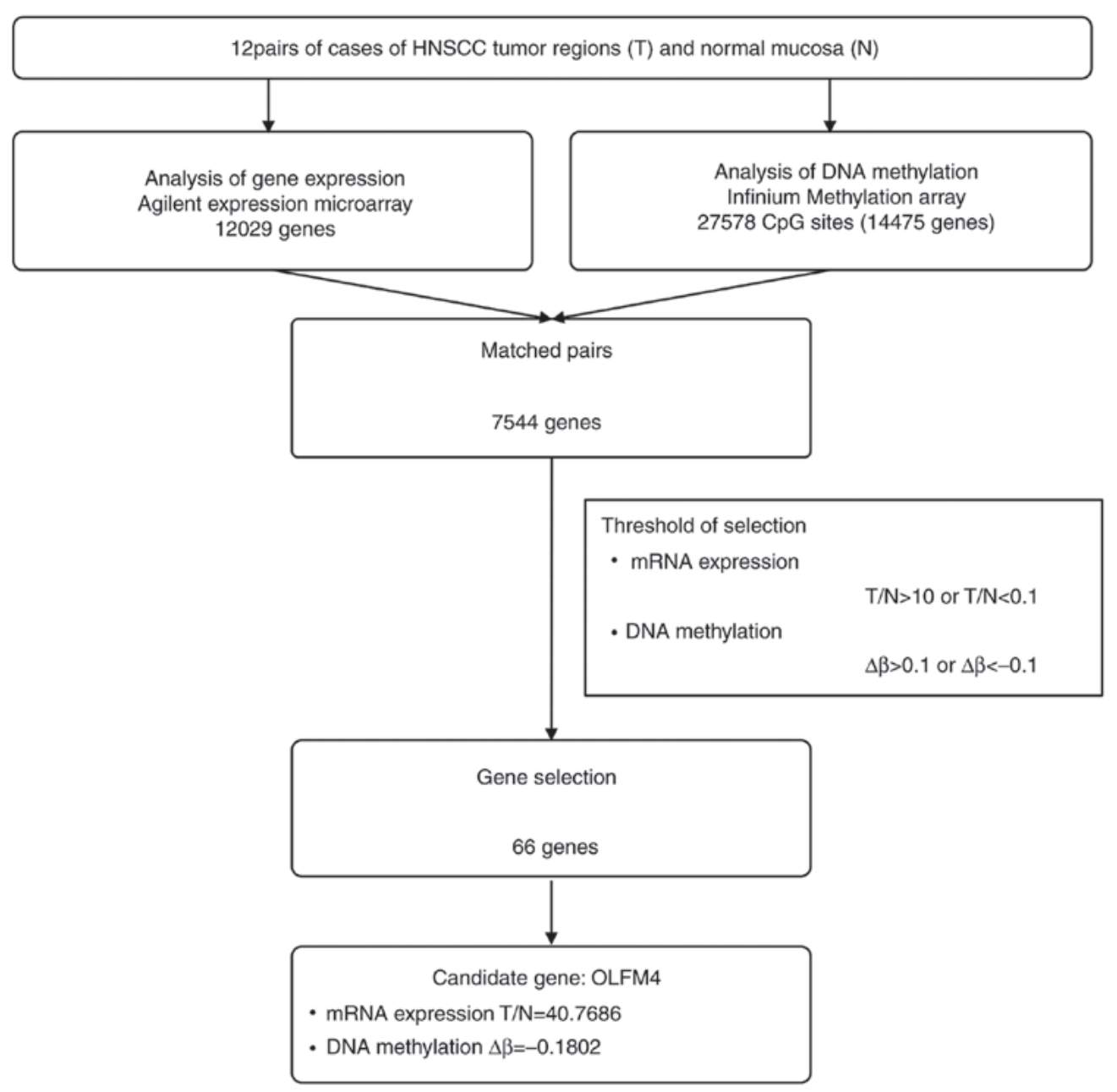

Figure 1. Gene selection flowchart in integrative screening analysis of mRNA expression and aberrant methylation with microarrays. The threshold of selection was set up as a flowchart, and OLFM4 was selected among 66 candidate genes that were suggested to be up/downregulated via altered DNA methylation in 12 tumor and non-tumor pairs of oral squamous cell carcinoma samples. OLFM4, olfactomedin 4; HNSCC, head and neck squamous cell carcinoma.

tissue using the QIAamp DNA Mini Kit (Qiagen), including on-column RNase digestion (Qiagen), as per the manufacturer's protocol. Bisulfite conversion of tissue genomic DNA (500 ng) was performed using the EZ DNA Gold methylation kit (Zymo Research Inc. A). Normalized M-values were generated using the R package HumMeth27KQCReport function, including the $\mathrm{X}$ chromosome data and using an average probe $\mathrm{P}$-value of 0.03 as the cutoff for sample inclusion. Individual BeadChip controls (DNA sample-dependent and sample-independent) confirmed efficient bisulfite conversion of DNA, hybridization specificity, base extension and target removal for all genomic DNA samples. A complete description of these controls is available from the manufacturer. Chromosome locations, RefSeq and GenBank accession numbers were retrieved from the National Center for Biotechnology Information build 36 (http://www.ncbi.nlm.nih.gov/mapview/stats/BuildStats. cgi?taxid=9606\&build $=36 \&$ ver $=1$ ).

Integration and validation of oligonucleotide and methylation microarray data. Two microarray data sets were integrated based on each gene symbol; a total of 7,544 matched gene pairs were identified using Microsoft Excel for Mac. For all gene pairs, $\mathrm{T} / \mathrm{N}$ and $\Delta \beta$ were calculated and selected according to the thresholds, and classified into four groups as follows:
Group $\mathrm{A}[\mathrm{T} / \mathrm{N}<0.1$ and $\Delta \beta>0.1]$, group $\mathrm{B}[\mathrm{T} / \mathrm{N}>10$ and $\Delta \beta>0.1]$, group $\mathrm{C}[\mathrm{T} / \mathrm{N}>10$ and $\Delta \beta<-0.1]$, and group $\mathrm{D}[\mathrm{T} / \mathrm{N}<0.1$ and $\Delta \beta<-0.1]$. The UniProt protein database was used as reference for the function of encoded proteins. The threshold for selecting candidate genes suggested to be strongly regulated via altered DNA methylation was set as $\mathrm{T} / \mathrm{N}>20$ and $\Delta \beta>0.15$ or $<-0.15$.

Cell culture with 5-azacytidine (5-aza). An inhibitor of DNA methyltransferases, 5-aza, was used for DNA demethylation in HNSCC cell lines. FaDu/HTB-43 (26) and Detroit 562/CCL-138 (27) were obtained from the American Type Culture Collection and cultured with 5-aza. Prior to this analysis, 11 cell lines, including CCL-138 and HTB-43, were cultured with 5-aza, and reverse transcription-quantitative polymerase chain reaction (RT-qPCR) analysis was performed to evaluate the OLFM4 expression (data not shown). Only CCL-138 and HTB-43 cells exhibited increased expression of OLFM4 following demethylation; therefore, these two cell lines were selected as they were considered suitable to substantiate the correlation between DNA methylation and OLFM4 expression.

OLFM4 expression was also analyzed in a normal fibroblast strain with/without demethylation treatment in 
RT-qPCR; however, the expression of OLFM4 in fibroblasts was extremely low and was not upregulated after demethylation treatment. Similarly, ISH was also performed in the fibroblast strain, and mRNA expression was very low, similar to the level in normal tissue adjacent to the tumor. Therefore, comparison with normal cells was not available (data not shown). Non-cancerous cells were not considered suitable as the study control.

HTB-43 and CCL-138 cells were cultured in DMEM (Nacalai Tesque, Inc.) with 10\% fetal bovine serum (Gibco; Thermo Fisher Scientific, Inc.), penicillin (100 U/ml) and streptomycin $(100 \mu \mathrm{g} / \mathrm{ml})$ at $37^{\circ} \mathrm{C}$ in a $5 \% \mathrm{CO}_{2}$ humidified atmosphere. The cell density was adjusted to $1 \times 10^{6}$ cells $/ 100-\mathrm{mm}$ dish $24 \mathrm{~h}$ prior to treatment. Stock solutions of 5-aza (Sigma-Aldrich; Merck KGaA) were dissolved in DMEM to concentrations of 0 (negative control), 0.2 and $2 \mu \mathrm{M}$. Cells were treated with 5-aza for 5 days, as previously reported (28).

RT-qPCR analysis. Total RNA was extracted from cultured cell lines using TRIzol reagent (Invitrogen; Thermo Fisher Scientific, Inc.) and the RNeasy mini kit (Qiagen), according to the manufacturer's instructions. Total RNA was reverse-transcribed into cDNA using the SuperScript IV VILO Master Mix kit (Invitrogen; Thermo Fisher Scientific, Inc.), as recommended by the manufacturer. The levels of mRNA expression for the OLFM4 gene were analyzed using custom TaqMan Expression Assays on the 7500 Fast Real-Time PCR System (Thermo Fisher Scientific, Inc.) employing the relative standard curve method. The probes and PCR primer sets employed were TaqMan Fast Advansed Master Mix and TaqMan gene expression assays (OLFM4 Hs00197437_m1; Applied Biosystems; Thermo Fisher Scientific, Inc.). GAPDH served as the endogenous control. Experiments were performed in triplicate, and the mean value for the three experiments was used as the quantification cycle $(\mathrm{Cq})$ value. All $\mathrm{Cq}$ values were normalized to that of GAPDH (GAPDH Hs0275899_g1; Applied Biosystems; Thermo Fisher Scientific, Inc.) in the same sample. The amplification program was according to the manufacturer's recommendations $\left(95^{\circ} \mathrm{C}\right.$ for $30 \mathrm{sec}$, followed by 40 cycles at $95^{\circ} \mathrm{C}$ for $3 \mathrm{sec}$ and at $60^{\circ} \mathrm{C}$ for $\left.30 \mathrm{sec}\right)$. The data were analyzed with the 7500 system SDS (version 1.4) software (Applied Biosystems; Thermo Fisher Scientific, Inc.).

Oligonucleotide microarray analysis for cell lines. Total RNA was extracted as described above. Oligonucleotide microarray analysis was conducted using Sure Print G3 Human Gene Expression 8x60K v3 Microarray (Agilent Technologies, Inc.) according to the manufacturer's protocol. The slides were scanned on the Agilent Sure Scan Microarray Scanner (G2600D; Agilent Technologies, Inc.) using one color scan setting for $8 \times 60 \mathrm{k}$ array slides. The scanned images were analyzed with Feature Extraction Software 11.5.1.1 (Agilent Technologies, Inc.) using default parameters to obtain background subtracted and spatially detrended Processed Signal intensities.

Statistical analysis. Statistical analysis was performed using SPSS software, version 23 (IBM Corp.). The correlations between OLFM4 positivity and clinicopathological parameters were assessed using the Chi-squared test. Between-group comparisons of the qPCR data were performed using Kruskal-Wallis test and Bonferroni correction as the post hoc test. Survival curves were constructed using the Kaplan-Meier method, and the log-rank test was used to compare groups. Survival time was calculated from the date of diagnosis to the date of the last follow-up visit or to the date of death. Statistical significance was set at $\mathrm{P}<0.05$.

\section{Results}

Fluctuation of mRNA expression and DNA methylation between HNSCC and normal mucosa. The mRNA expression ratio $(\mathrm{T} / \mathrm{N})$ and DNA methylation $(\Delta \beta)$ of 12 clinical sample pairs were analyzed using expression microarray and methylation microarray. The mean of the $\mathrm{T} / \mathrm{N}$ and $\Delta \beta$ data from the 12 samples was calculated for each gene. There were 7,544 matched gene pairs, and thresholds for the two categories were set up as follows: $\mathrm{T} / \mathrm{N}>10$ or $<0.1$ and $\Delta \beta$ $>0.1$ or $<-0.1$. Based on these threshold values, 66 genes were extracted (Fig. 1) and assigned into four groups based on the levels of $\mathrm{T} / \mathrm{N}$ and $\Delta \beta$ as follows: Group $\mathrm{A}[\mathrm{T} / \mathrm{N}<0.1$ and $\Delta \beta>0.1$, five genes], group $\mathrm{B}$ [T/N>10 and $\Delta \beta>0.1,22$ genes], group $\mathrm{C}[\mathrm{T} / \mathrm{N}>10$ and $\Delta \beta<-0.1,39$ genes], and group $\mathrm{D}$ $[\mathrm{T} / \mathrm{N}<0.1$ and $\Delta \beta<-0.1$, no genes]. Each group included genes suggested to be regulated by methylation. Incidentally, a small number of cancer prognostic genes were included in these groups (representative genes are shown in Table I; all data are included in Table SI).

Next, we validated individual genes in each group. Based on the function of the encoded protein, clustering was performed and indicated the functional tendency in each group. In group $\mathrm{C}$, exhibiting low DNA methylation $(\Delta \beta)$ and high mRNA expression ( $\mathrm{T} / \mathrm{N})$, 'immune/inflammation' genes were included most frequently (14/39 genes, respectively; $35.9 \%$ of group C). Interestingly, this tendency is different in each group; group A contained the most 'growth factor' members (2/5 genes, $40 \%)$, and group B included the most 'signal' members (8/22 genes, 36.4\%) (Fig. 2, lower panel). The methylation status in clinical samples used for ISH and IHC was not analyzed.

Upregulation of gene expression in HNSCC and selection of candidate genes. From these four gene groups, OLFM4 was selected as a candidate gene regulated by DNA methylation. OLFM4 was highly expressed, with a T/N ratio of 40.7686 and low methylation (-18.02\%), suggesting that OLFM4 was overexpressed by promoter hypomethylation in HNSCC.

OLFM4 is a stem cell-related gene in intestinal crypt cells (29), and was included in group C. OLFM4 encodes a secreted protein that is implicated in cell-cell adhesion. We hypothesized that OLFM4 was correlated with carcinogenesis via its stemness and function; therefore, it was selected as a candidate gene.

Demethylation treatment recovered OLFM4 expression in HNSCC cell lines. The CCL-138 and HTB-43 cell lines were cultured with 5-aza. As the clinical samples used in the microarray could not provide the required amount for experiments, qPCR was not performed. Therefore, experiments were conducted using cell lines as a substitute. 
Table I. Genes regulated by altered DNA methylation in tumor tissue (T) compared with normal adjacent normal mucosa (N).

\begin{tabular}{|c|c|c|c|}
\hline $\begin{array}{l}\text { Gene } \\
\text { symbol }\end{array}$ & $\begin{array}{l}\text { mRNA expression } \\
\text { ratio }(\mathrm{T} / \mathrm{N})\end{array}$ & $\begin{array}{c}\text { DNA } \\
\text { methylation }(\Delta \beta)\end{array}$ & Description \\
\hline IGLL1 & 136.4124102143 & -0.171246308 & Immunoglobulin lambda-like polypeptide 1 , transcript variant 1 \\
\hline ACTAl & 76.7808690487 & 0.241393352 & Actin, alpha 1, skeletal muscle \\
\hline TNFRSF 17 & 71.2474598502 & 0.183141233 & Tumor necrosis factor receptor superfamily, member 17 \\
\hline OLFM4 & 40.7686369333 & -0.180180495 & Olfactomedin 4 \\
\hline MMP11 & 32.4300605647 & 0.159569173 & Matrix metallopeptidase 11 (stromelysin 3) \\
\hline ACTN2 & 30.1225359676 & 0.269785628 & Actinin, alpha 2 \\
\hline MYH7 & 24.8015332005 & -0.186027005 & Myosin, heavy chain 7 , cardiac muscle, beta \\
\hline SOX11 & 22.5603549704 & 0.251592997 & SRY (sex determining region Y)-box 11 \\
\hline BST2 & 20.3898517378 & -0.348478699 & Bone marrow stromal cell antigen 2 \\
\hline MMP13 & 250.9824673732 & -0.111671958 & Matrix metallopeptidase 13 (collagenase 3) \\
\hline CXCL13 & 135.1941882092 & 0.131270067 & Chemokine (C-X-C motif) ligand 13 (B-cell chemoattractant) \\
\hline LHX2 & 22.183761883 & 0.110752037 & LIM homeobox 2 \\
\hline$M A G E A 1$ & 18.4626044 & -0.121956831 & Melanoma antigen family A, 1 (directs expression of antigen MZ2-E) \\
\hline$D K K 1$ & 16.92536015 & -0.126459913 & Dickkopf homolog 1 (Xenopus laevis) \\
\hline$P I K 3 R 5$ & 14.495631 & -0.10109523 & Phosphoinositide-3-kinase, regulatory subunit 5 \\
\hline CCL7 & 13.79784112 & 0.117473304 & Chemokine (C-C motif) ligand 7 \\
\hline
\end{tabular}

The mRNA expression ratio $(\mathrm{T} / \mathrm{N})$ and DNA methylation score $(\Delta \beta)$ were calculated as expression score in tumor tissue $(\mathrm{T}) /$ normal tissue $(\mathrm{N})$, and as methylation score in tumor tissue $(\mathrm{T})$-normal tissue $(\mathrm{N})$, respectively. When the selection thresholds for the two data categories were set up as $\mathrm{T} / \mathrm{N}>20$ and $\Delta \beta>0.15$ or $<-0.15,9$ genes were selected (first 9 genes, including $O L F M 4$ ). Certain cancer prognostic genes were also picked up (selection threshold: $\mathrm{T} / \mathrm{N}>10$ and $\Delta \beta>0.1$ or $<-0.1$ (last 7 genes, only representative genes are shown; all genes are available in Table SI). OLFM4, olfactomedin 4.

RT-qPCR was performed to verify the regulation of OLFM4 gene expression by DNA methylation. In CCL-138, OLFM4 expression was increased by 5 -aza in a concentration-dependent manner. At 0.2 and $2 \mu \mathrm{M} 5$-aza, the expression of OLFM4 was 3.49 and 9.82 times higher, respectively, compared with the control. In HTB-43, the recovery of OLFM4 expression was dependent on 5-aza concentration (Fig. 3A). However, statistical significance was only observed for CCL-138 cells (Kruskal-Wallis test, $\mathrm{P}=0.044)$. In Bonferroni correction, a statistically significant difference was only observed between 0 (negative) and $2 \mu \mathrm{M}$ in CCL-138 cells.

Similarly, in the oligonucleotide microarray, the two cell lines exhibited a concentration-dependent upregulation of OLFM4. However, in this experiment, sufficient amount of mRNA was not collected, and statistical analysis was not performed due to the insufficient sample size ( $\mathrm{n}=1$, data not shown).

OLFM4 mRNA and protein expression in tumor lesions. RNA ISH assay revealed that OLFM4 mRNA was diffusely expressed in HNSCC cells; however, the basal layer cells were not positive (Fig. 3B-a, HE staining and B-b, ISH). To confirm the overexpression of the OLFM4 protein, IHC staining was performed using TMA. The OLFM4 protein was found to be diffusely expressed in the tumor (Fig. 3B-c). The results of IHC staining demonstrated that the OLFM4 protein was expressed in $47.5 \%$ (28/59) of the samples. The OLFM4 protein was expressed in the cytoplasm of tumor cells (Fig. 3B-c). The staining scores were classified as $2+/ 1+/ 0$ (negative); $2+$ was observed in only one case. The results of IHC staining and ISH positivity and negativity were similar. However, in ISH, the result was evaluated as + or -, as the signal tended to be weak. Scoring by expression intensity was only performed in IHC. In this analysis, ISH was used to confirm the expression of OLFM4, not only at the protein but also at the mRNA level. In normal mucosa and intratumor fibroblasts, excluding muscle fibers, both IHC and ISH were negative (Fig. 3B-d and B-e).

No statistically significant association between OLFM4 protein expression and sex or clinical stage was observed. A trend was observed between positive OLFM4 protein expression and poor tumor differentiation; however, it was not statistically significant $(\mathrm{P}=0.06)$ (Table II).

The median age of the 59 patients ( 36 men and 23 women) who had tongue $\mathrm{SCC}<4 \mathrm{~cm}$ in diameter (classified as T2, T1, and Tis, N0, M0) in the surgery alone group was 60 years (range, 28-84 years). During a median follow-up of 2,047 days (range, 219-3,956 days), the overall 5-year survival rate was $64.2 \%$, with a median survival of 2,047 days [95\% confidence interval (CI): 1,720-2,372 days]. Of the 59 cases of tongue SCC, $28(47.5 \%)$ were positive and 31 (52.5\%) were negative for OLFM4 expression. $\chi^{2}$ and log-rank analyses revealed no significant difference between OLFM4-positive (median survival: 1,111 days; $95 \%$ CI: 1,802-3,038 days) and -negative tumors (median survival: 2,109 days; 95\% CI: 2249-3038 days; $\mathrm{P}=0.34$; Fig. 3C).

\section{Discussion}

The present study focused on the regulatory system of gene expression via aberrant DNA methylation in HNSCC. From 


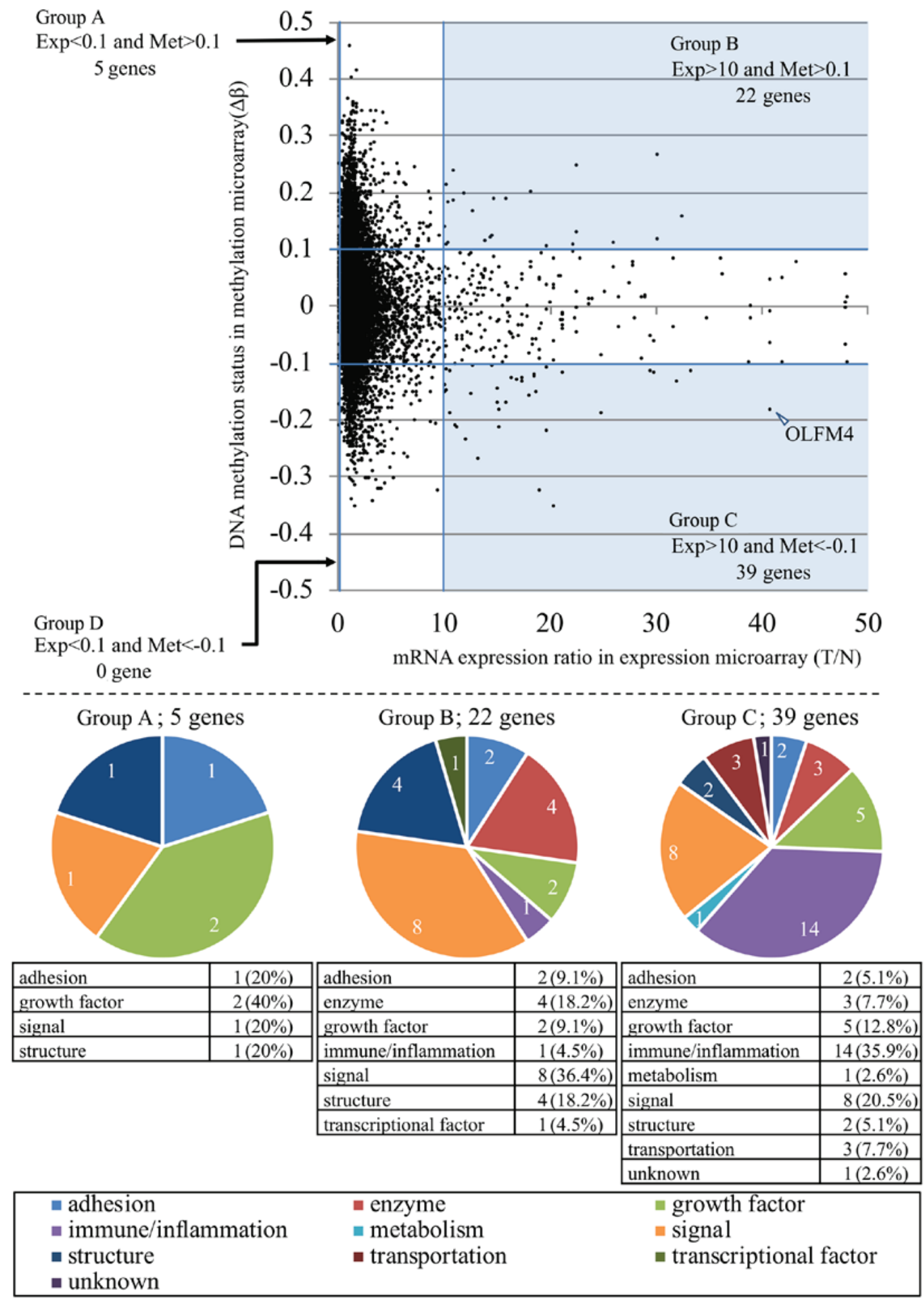

Figure 2. Correlation between mRNA expression (T/N ratio) and DNA methylation $(\Delta \beta)$. Blue area shows the four groups that were created by thresholds for the two categories as follows: $\mathrm{T} / \mathrm{N}>10$ or $<0.1$ and $\Delta \beta>0.1$ or $<-0.1$. OLFM4 was included in group C, exhibiting higher mRNA expression and lower DNA methylation. Functional clustering of the 66 genes in four groups is shown in the graphs. The complete gene list is available in supplementary materials (Table SI). T/N, tumor/non-tumor; OLFM4, olfactomedin 4.

integrated screening, OLFM4, which was upregulated by promoter hypomethylation, was selected. We previously reported on the regulatory system of stemness molecules, Bmi-1 and HMGA1, in early-stage HNSCC (30). CSCs, which are undifferentiated, pluripotent cells with self-renewal ability, give rise to other malignant daughter cells and are considered to be correlated with tumor metastasis and drug resistance. In addition, epigenetics is deeply involved in the drug resistance exhibited by CSCs. CSCs are resistant to conventional chemotherapy, and can re-establish the tumor 
A

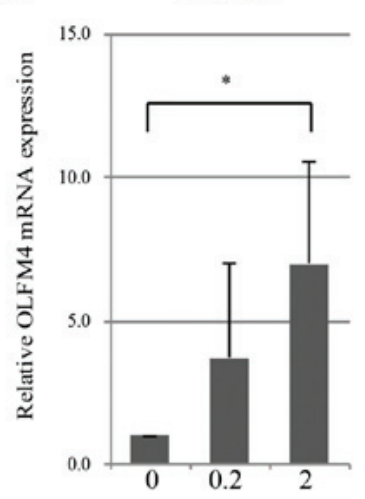

5-Aza. concentration $(\mu \mathrm{M})$

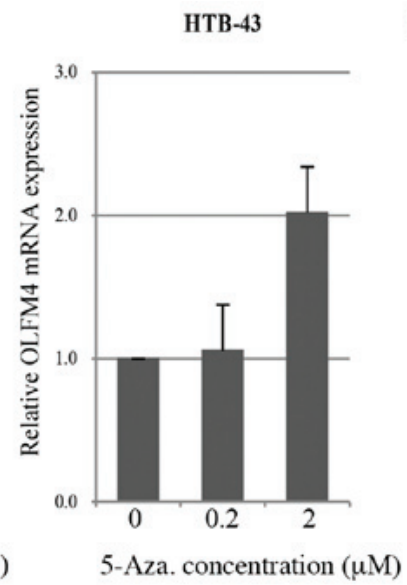

5-Aza. concentration $(\mu \mathrm{M})$
C

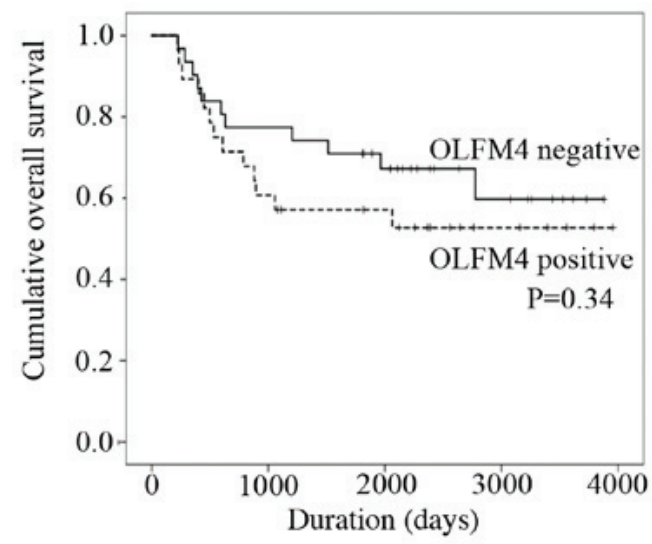

B
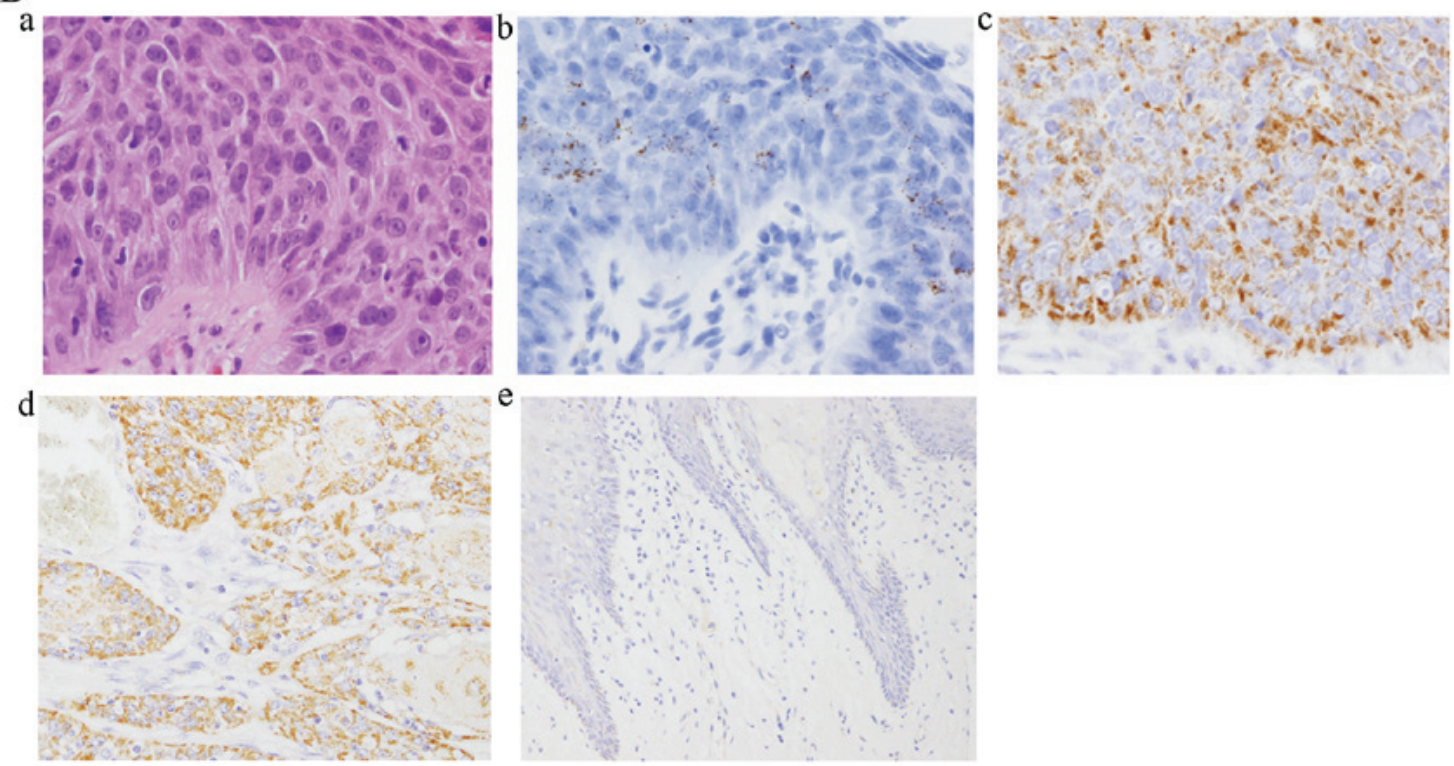

Figure 3. (A) OLFM4 mRNA expression by RT-qPCR in demethylation-treated HNSCC cell lines (n=3). OLFM4 mRNA expression levels were increased in a concentration-dependent manner by the demethylating agent 5-azacytidine (5-aza) in CCL-138 and HTB-43 cells. Statistical analysis was performed using Kruskal-Wallis test. Both cell lines exhibited 5-aza concentration-depending upregulation of OLFM4; however, statistical significance was observed in CCl-138 ( $\mathrm{P}=0.044)$, but not in HTB-43 cells $(\mathrm{P}=0.055)$. Using Bonferroni correction as a post hoc test, statistical significance was only observed between 0 (negative) and $2 \mu \mathrm{M} 5$-aza in the CCL-138 cell line. Error bars indicate mean \pm standard deviation. ${ }^{*} \mathrm{P}=0.038$ in Bonferroni correction. (B) $O L F M 4$ expression determined by in situ hybridization (ISH) and immunohistochemistry (IHC). Well-differentiated (non-invasive) SCC: a) HE, b) ISH and c) IHC); d) poorly differentiated SCC; e) normal adjacent squamous epithelium. (C) Cumulative overall survival analysis in patients with tongue SCC. Patients with OLFM4 expression (scored as 2+ and 1+) are shown by the dotted line and patients with no OLFM4 expression (scored as negative) are shown by the solid line. The OLFM4-positive group had a worse survival outcome compared with the negative group, but the difference was not statistically significant $(\mathrm{P}=0.34)$ in the log-rank test. OLFM4, olfactomedin 4; HNSCC, head and neck squamous cell carcinoma; RT-qPCR, reverse transcription-quantitative polymerase chain reaction; $\mathrm{HE}$, hematoxylin and eosin.

at locoregional or distant sites, make treatment difficult. Therefore, a better understanding of the regulatory system of CSCs may lead to the development of new therapeutic strategies (31). The present study focused on the correlation between DNA methylation and OLFM4, which is known as a robust marker for human intestinal stem cells (29). OLFM4 has been widely used as a stem cell marker for murine small intestine (32). ISH staining (29,33) and IHC (34,35) studies revealed that $O L F M 4$-positive cells located at the crypt base co-expressed Lgr5 in the small intestine. Therefore, OLFM4 was selected as a potential marker for stemness expressed in HNSCC.

A previous study reported OLFM4 expression in oral $\mathrm{SCC}$, and IHC staining revealed overexpression of OLFM4 in $75 \%$ of 76 HNSCC cases tested (36). However, the regulatory mechanism of OLFM4 in HNSCC has not been investigated. OLFM4 was confirmed to be hypomethylated and upregulated in oral SCC when compared with the surrounding normal tissue. RT-qPCR demonstrated that demethylation treatment induced overexpression of OLFM4. The experiment was performed in triplicate, and statistical significance was observed in CCL-138 cells. Although not statistically verified, the microarray results for demethylated cell lines also demonstrated that hypomethylation induced OLFM4 upregulation, supporting the result of the RT-qPCR analysis. To the best of the authors' knowledge, this is the first study to report that promoter methylation regulates OLFM4 expression in HNSCC. 
Table II. Characteristics of 59 squamous cell carcinomas based on immunostaining.

\begin{tabular}{|c|c|c|c|}
\hline \multirow[b]{2}{*}{ Characteristics } & \multicolumn{2}{|c|}{ OLFM4 expression } & \multirow[b]{2}{*}{ P-value } \\
\hline & $2+/ 1+$ & - & \\
\hline Number of tumors & 28 & 31 & NA \\
\hline Mean age (years) \pm standard deviation $^{\mathrm{a}}$ & $58.3 \pm 13.16$ & $63.0 \pm 26.33$ & NA \\
\hline Sex & & & 0.12 \\
\hline Female & 8 & 15 & \\
\hline Male & 20 & 16 & \\
\hline Differentiation & & & 0.06 \\
\hline Well-differentiated $(n=40)$ & 15 & 25 & \\
\hline Moderately differentiated $(n=7)$ & 4 & 3 & \\
\hline Poorly differentiated $(n=12)$ & 9 & 3 & \\
\hline Stage & & & 0.62 \\
\hline I & 27 & 29 & \\
\hline II & 1 & 2 & \\
\hline
\end{tabular}

${ }^{\mathrm{a}}$ All 59 cases: Mean age \pm standard deviation, $60.1 \pm 12.87$. Statistical analysis was performed using Chi-squared test to evaluate the association between OLFM4 expression and clinical variables. There was no statistical significance in these 59 clinical samples; a trend was observed between positive OLFM4 protein expression and poor tumor differentiation; however, it was not statistically significant ( $\mathrm{P}=0.06)$. OLFM4, olfactomedin 4; NA, not applicable.

OLFM4 has been reported to be involved in various biological processes, such as cell-to-cell interaction, apoptosis, migration and cell cycle regulation, in different types of cancers (37). Aberrant overexpression of OLFM4 has been observed in certain cancerous lesions, particularly in cancers of the digestive tract, including gastric $(4,5)$, pancreatic (6) and colon cancers $(38,39)$. In addition, OLFM4 has been reported as a novel biomarker for the differentiation and progression of gastrointestinal cancer (4). Although the mechanism regulating OLFM4 expression has only been shown in certain types of cancer, such as gastric (40) and colorectal cancer (34), it has been revealed that the expression of OLFM4 is associated with promoter methylation status. However, the detailed mechanisms underlying the role of OLFM4 in cancer remain unclear. Oue et al reported that the expression of OLFM4 in gastric cancer tissues is observed more frequently in stage I/II compared with stage III/IV cancers on immunostaining. In addition, serum OLFM4 concentration in preoperative gastric cancer patients was higher compared with that in healthy individuals (4). Downregulation of OLFM4 expression in advanced tumors is associated with decreased patient survival (5). This suggests that OLFM4 not only plays a role in the early stages of tumor initiation, but also exerts an inhibitory effect on cancer cell invasion and metastases in the advanced stages of tumor development (41). It has been previously reported that the downregulation of OLFM4 expression is induced by hypermethylation in advanced gastric cancer (41). Our results were consistent with the findings of Guo et al (41), in that promoter methylation regulates the expression of OLFM4. OLFM4 may exert a cancer-promoting effect via apoptosis inhibition during the early stages, suggesting that OLFM4 inactivation may be crucial for tumor progression or metastasis (42). By contrast, OLFM4 was found to be highly expressed in normal prostate tissue, moderately expressed in benign prostatic hyperplasia tissues, and not expressed in prostate cancer tissues, indicating that OLFM4 acted as a tumor-suppressing gene (7).

OLFM4 expression is enhanced in more highly differentiated gastric and colon cancers, and is markedly reduced or absent in poorly differentiated or undifferentiated cancers $(11,34)$. In the present study, OLFM4 tended to be expressed more frequently in poorly differentiated tumors and cases with poor prognosis. Although no statistical significance was established, the cumulative overall survival rate tended to be worse in OLFM4-positive cases. Therefore, OLFM4 cannot be considered an independent prognostic marker for HNSCC. In addition, no association was found between OLFM4 expression and TNM stage of HNSCC. These results are inconsistent with those of previous reports on other cancers. Takadate et al reported that OLFM4 expression was correlated with poor prognosis in pancreatic ductal adenocarcinoma (PDAC) (43). In PDAC cells, OLFM4 mRNA was highly expressed during the $\mathrm{S}$ phase of the cell cycle, and the cell cycle was arrested at the $\mathrm{S}$ phase by the downregulation of OLFM4 mRNA expression. This finding demonstrated that OLFM4 promotes proliferation of PDAC cells by favoring transition from the $\mathrm{S}$ to the $\mathrm{G} 2 / \mathrm{M}$ phase. Thus, the expression and function of $O L F M 4$ in carcinogenesis is organ-selective and limited by tumor size. These results reported for PDAC are in agreement with our results, and indicate that OLFM4 has a similar function in HNSCC.

The findings of the present study indicate that DNA methylation reduces the expression of the stemness molecule OLFM4, and it may affect cell heterogeneity, but does not affect prognosis. However, DNA methylation occurs prior to carcinogenesis in normal tissue, and OLFM4 expression would only be 
involved in tumor initiation in the very early stages of carcinogenesis, and not in progression. Thus, OLFM4 expression may not affect HNSCC prognosis. This is similar to our previous report on Bmil (30), which tends to be expressed in early and well-differentiated cancers, and is lost in advanced/poorly differentiated HNSCCs. Thus, it was hypothesized that Bmil expression arises in the cancer-developing stage of early tumors with high plasticity. The long half-life of the cancer 'cell of origin' allows the accumulation of multiple mutations and epigenetic changes required for multi-step evolution toward progression. These progressed cancer cells exhibited decreased Bmil expression and gained proliferative activity instead of loss of plasticity (30). Hence, it was concluded that Bmil does not affect the prognosis of HNSCC. As OLFM4 is only expressed during the developing stage of early tumors, it may not contribute to HNSCC prognosis. There was tendency of correlation with poor differentiation in clinicopathological characteristics, but was not statistically significant $(\mathrm{P}=0.06)$. However, we only analyzed data from 59 cases and had no available clinical samples. This requires further investigation by analyzing more samples.

The integrated screening analysis provided interesting data. Some genes that are reported as a prognostic factors in other cancers also exhibited fluctuations of $\mathrm{T} / \mathrm{N}$ and $\Delta \beta$ (Table I). It has been reported that the expression of melanoma antigen encoding gene 1 (MAGEA1) was correlated with prognosis in differentiated advanced gastric cancer (44) and ovarian cancer (45). Dickkopf-1 (DKK1) was also found to be correlated with the prognosis of breast cancer (46), laryngeal SCC (47), non-small-cell lung cancer (48), chondrosarcoma (49), hepatocellular carcinoma $(50,51)$ and cervical cancer $(52)$. However, the regulatory mechanism of these genes based on DNA methylation in HNSCC has not been investigated. Further studies are required to identify their potential as novel biomarkers or prognostic markers.

In conclusion, the aberrant stemness gene expression caused by altered DNA methylation and its involvement in early HNSCC characteristics was investigated, and the results revealed a correlation with OLFM4 expression via promoter methylation and tumor cell heterogeneity in HNSCC.

\section{Acknowledgements}

The authors would like to thank Ms. Sachiko Miura, Ms. Toshiko Sakaguchi, Ms. Chizu Kina, Mr. Hideo Tsukamoto and Mr. Tadayuki Sato for their skillful technical assistance.

\section{Funding}

The present study was supported in part by a Grant-in-Aid for Scientific Research JSPS KAKENHI (grant no. 17K08710) from the Ministry of Education, Culture, Sports, Science and Technology; partial support was received from the Research and Study Program of Tokai University Educational System General Research Organization.

\section{Availability of data and materials}

The datasets used and/or analyzed during the present study are available from the corresponding author on reasonable request.

\section{Authors' contribution}

TM conceived this project; TS and TM designed the experiments and wrote the manuscript; TS, HY, ER KH and TM performed the experiments and bioinformatics analysis; TS, HY, AK, YO and TM provided clinical samples and designed the study. All authors have read and approved the final version of this manuscript for publication.

\section{Ethics approval and consent to participate}

Informed consent was obtained from the patients and the Ethics Committee of the National Cancer Center Hospital (Tokyo, Japan) approved the procedures (approval no. 2010-077).

\section{Patient consent for publication}

Consent for publication was obtained from all patients included in this study.

\section{Competing interests}

The authors declare that they have no competing interests.

\section{References}

1. Ferlay J, Soerjomataram I, Dikshit R, Eser S, Mathers C Rebelo M, Parkin DM, Forman D and Bray F: Cancer incidence and mortality worldwide: Sources, methods and major patterns in GLOBOCAN 2012. Int J Cancer 136: E359-E386, 2015.

2. Patel SS, Shah KA, Shah MJ, Kothari KC and Rawal RM: Cancer stem cells and stemness markers in oral squamous cell carcinomas. Asian Pac J Cancer Prev 15: 8549-8556, 2014.

3. Zhang J, Liu WL, Tang DC, Chen L, Wang M, Pack SD, Zhuang Z and Rodgers GP: Identification and characterization of a novel member of olfactomedin-related protein family, hGC-1, expressed during myeloid lineage development. Gene 283: 83-93, 2002.

4. Oue N, Sentani K, Noguchi T, Ohara S, Sakamoto N, Hayashi T, Anami K, Motoshita J, Ito M, Tanaka S, et al: Serum olfactomedin 4 (GW112, hGC-1) in combination with Reg IV is a highly sensitive biomarker for gastric cancer patients. Int J Cancer 125 : 2383-2392, 2009.

5. Luo Z, Zhang Q, Zhao Z, Li B, Chen J and Wang Y: OLFM4 is associated with lymph node metastasis and poor prognosis in patients with gastric cancer. J Cancer Res Clin Oncol 137: 1713-1720, 2011.

6. Kobayashi D, Koshida S, Moriai R, Tsuji N and Watanabe N: Olfactomedin 4 promotes $\mathrm{S}$-phase transition in proliferation of pancreatic cancer cells. Cancer Sci 98: 334-340, 2007.

7. Chen L, Li H, Liu W, Zhu J, Zhao X, Wright E, Cao L, Ding I and Rodgers GP: Olfactomedin 4 suppresses prostate cancer cell growth and metastasis via negative interaction with cathepsin D and SDF-1. Carcinogenesis 32: 986-994, 2011.

8. Li H, Liu W, Chen W, Zhu J, Deng CX and Rodgers GP: Olfactomedin 4 deficiency promotes prostate neoplastic progression and is associated with upregulation of the hedgehog-signaling pathway. Sci Rep 5: 16974, 2015.

9. Kulkarni NH, Karavanich CA, Atchley WR and Anholt RR: Characterization and differential expression of a human gene family of olfactomedin-related proteins. Genet Res 76: 41-50, 2000.

10. Liu RH, Yang MH, Xiang H, Bao LM, Yang HA, Yue LW, Jiang X, Ang N, Wu LY and Huang Y: Depletion of OLFM4 gene inhibits cell growth and increases sensitization to hydrogen peroxide and tumor necrosis factor-alpha induced-apoptosis in gastric cancer cells. J Biomed Sci 19: 38, 2012.

11. Liu W, Zhu J, Cao L and Rodgers GP: Expression of hGC-1 is correlated with differentiation of gastric carcinoma. Histopathology 51: 157-165, 2007. 
12. Besson D, Pavageau AH, Valo I, Bourreau A, Bélanger A, Eymerit-Morin C, Moulière A, Chassevent A, Boisdron-Celle M, Morel A, et al: A quantitative proteomic approach of the different stages of colorectal cancer establishes OLFM4 as a new nonmetastatic tumor marker. Mol Cell Proteomics 10: M111.009712, 2011.

13. Zhang X, Huang Q, Yang Z, Li Y and Li CY: GW112, a novel antiapoptotic protein that promotes tumor growth. Cancer Res 64: 2474-2481, 2004

14. Mitani Y, Oue N, Matsumura S, Yoshida K, Noguchi T, Ito M, Tanaka S, Kuniyasu H, Kamata N and Yasui W: Reg IV is a serum biomarker for gastric cancer patients and predicts response to 5-fluorouracil-based chemotherapy. Oncogene 26: 4383-4393, 2007.

15. Sun YW, Chen KM, Imamura Kawasawa Y, Salzberg AC, Cooper TK, Caruso C, Aliaga C, Zhu J, Gowda K, Amin S and El-Bayoumy K: Hypomethylated Fgf3 is a potential biomarker for early detection of oral cancer in mice treated with the tobacco carcinogen dibenzo[def,p]chrysene. PLoS One 12: e0186873, 2017.

16. Umair A, Tarakji B, Ibrahim A, Nasser A, Fq A and Shreen A: Quantitative study of epigenetic signature in head and neck squamous cell carcinoma. Turk J Med Sci 45: 372-386, 2015.

17. Foy JP, Pickering CR, Papadimitrakopoulou VA, Jelinek J, Lin SH, William WN Jr, Frederick MJ, Wang J, Lang W, Feng L, et al: New DNA methylation markers and global DNA hypomethylation are associated with oral cancer development. Cancer Prev Res (Phila) 8: 1027-1035, 2015.

18. Bakhtiar SM, Ali A and Barh D: Epigenetics in head and neck cancer. Methods Mol Biol 1238: 751-769, 2015.

19. Prawdzic Senkowska A, Kiczmer P, Strzelczyk JK, Kowalski D, Krakowczyk $€$ and Ostrowska Z: Impact of HPV infection on gene expression and methylation in oral cancer patients. J Med Microbiol 68: 440-445, 2019

20. Zhou C, Shen Z, Ye D, Li Q, Deng H, Liu H and Li J: The Association and clinical significance of CDKN2A promoter methylation in head and neck squamous cell carcinoma: A meta-analysis. Cell Physiol Biochem 50: 868-882, 2018.

21. Mydlarz WK, Hennessey PT, Wang H, Carvalho AL and Califano JA: Serum biomarkers for detection of head and neck squamous cell carcinoma. Head Neck 38: 9-14, 2016.

22. Shi H, Chen X, Lu C, Gu C, Jiang H, Meng R, Niu X, Huang Y and $\mathrm{Lu} \mathrm{M}$ : Association between P16INK4a promoter methylation and HNSCC: A meta-analysis of 21 published studies. PLoS One 10: e0122302, 2015.

23. Zhao J, Shu P, Duan F, Wang X, Min L, Shen Z, Ruan Y, Qin J, Sun Y and Qin X: Loss of OLFM4 promotes tumor migration through inducing interleukin-8 expression and predicts lymph node metastasis in early gastric cancer. Oncogenesis 5: e234, 2016

24. Jang BG, Lee BL and Kim WH: Olfactomedin-related proteins 4 (OLFM4) expression is involved in early gastric carcinogenesis and of prognostic significance in advanced gastric cancer. Virchows Arch 467: 285-294, 2015.

25. Barnes L, Eveson J, Reichart P and Sidransky D: World Health Organization classifications tumours. Pathology and genetics of head and neck tumours. Lyon: IARC, 2005.

26. Rangan SR: A new human cell line (FaDu) from a hypopharyngeal carcinoma. Cancer 29: 117-121, 1972.

27. Peterson WD Jr, Stulberg CS, Swanborg NK and Robinson AR Glucose-6-phosphate dehydrogenase isoenzymes in human cell cultures determined by sucrose-agar gel and cellulose acetate zymograms. Proc Soc Exp Biol Med 128: 772-776, 1968.

28. Zhou Y and $\mathrm{Hu} \mathrm{Z}$ : Epigenetic DNA demethylation causes inner ear stem cell differentiation into hair cell-like cells. Front Cell Neurosci 10: 185, 2016

29. van der Flier LG, Haegebarth A, Stange DE, van de Wetering M and Clevers H: OLFM4 is a robust marker for stem cells in human intestine and marks a subset of colorectal cancer cells. Gastroenterology 137: 15-17, 2009.

30. Yamazaki H, Mori T, Yazawa M, Maeshima AM, Matsumoto F, Yoshimoto S, Ota Y, Kaneko A, Tsuda H and Kanai Y: Stem cell self-renewal factors Bmil and HMGA2 in head and neck squamous cell carcinoma: Clues for diagnosis. Lab Invest 93 1331-1338, 2013

31. Castilho RM, Squarize CH and Almeida LO: Epigenetic modifications and Head and neck cancer: Implications for tumor progression and resistance to therapy. Int J Mol Sci 18: pii: E1506, 2017.

32. Schuijers J, van der Flier LG, van Es J and Clevers H: Robust cre-mediated recombination in small intestinal stem cells utilizing the olfm4 locus. Stem Cell Reports 3: 234-241, 2014.
33. Ziskin JL, Dunlap D, Yaylaoglu M, Fodor IK, Forrest WF, Patel R, Ge N, Hutchins GG, Pine JK, Quirke P, et al: In situ validation of an intestinal stem cell signature in colorectal cancer. Gut 62: 1012-1023, 2013.

34. Liu W, Liu Y, Zhu J, Wright E, Ding I and Rodgers GP: Reduced hGC-1 protein expression is associated with malignant progression of colon carcinoma. Clin Cancer Res 14: 1041-1049, 2008.

35. Gersemann M, Becker S, Nuding S, Antoni L, Ott G, Fritz P, Oue N, Yasui W, Wehkamp J and Stange EF: Olfactomedin-4 is a glycoprotein secreted into mucus in active IBD. J Crohns Colitis 6: 425-434, 2012.

36. Marimuthu A, Chavan S, Sathe G, Sahasrabuddhe NA, Srikanth SM, Renuse S, Ahmad S, Radhakrishnan A, Barbhuiya MA, Kumar RV, et al: Identification of head and neck squamous cell carcinoma biomarker candidates through proteomic analysis of cancer cell secretome. Biochim Biophys Acta 1834: 2308-2316, 2013.

37. Grover PK, Hardingham JE and Cummins AG: Stem cell marker olfactomedin 4: Critical appraisal of its characteristics and role in tumorigenesis. Cancer Metastasis Rev 29: 761-775, 2010.

38. Liu W, Li H, Hong SH, Piszczek GP, Chen W and Rodgers GP: Olfactomedin 4 deletion induces colon adenocarcinoma in $\mathrm{Apc}^{\mathrm{Min} /+}$ mice. Oncogene 35: 5237-5247, 2016.

39. Sentani K, Sakamoto N, Shimamoto F, Anami K, Oue N and Yasui W: Expression of olfactomedin 4 and claudin-18 in serrated neoplasia of the colorectum: A characteristic pattern is associated with sessile serrated lesion. Histopathology 62: 1018-1027, 2013.

40. Yamanoi K, Arai E, Tian Y, Takahashi Y, Miyata S, Sasaki H, Chiwaki F, Ichikawa H, Sakamoto H, Kushima R, et al: Epigenetic clustering of gastric carcinomas based on DNA methylation profiles at the precancerous stage: Its correlation with tumor aggressiveness and patient outcome. Carcinogenesis 36: 509-520, 2015

41. Guo LL, He ZC, Yang CQ, Qiao PT and Yin GL: Epigenetic silencing of olfactomedin-4 enhances gastric cancer cell invasion via activation of focal adhesion kinase signaling. BMB Rep 48: 630-635, 2015 .

42. Su W, Luo L, Wu F, Lai Z, Li X, Xie Z, Tang Z, Yang Z and Liang R: Low expression of olfactomedin 4 correlates with poor prognosis in smoking patients with non-small cell lung cancer. Hum Pathol 46: 732-738, 2015.

43. Takadate T, Onogawa T, Fukuda T, Motoi F, Suzuki T, Fujii K, Kihara M, Mikami S, Bando Y, Maeda S, et al: Novel prognostic protein markers of resectable pancreatic cancer identified by coupled shotgun and targeted proteomics using formalin-fixed paraffin-embedded tissues. Int J Cancer 132: 1368-1382, 2013.

44. Ogata K, Aihara R, Mochiki E, Ogawa A, Yanai M, Toyomasu Y, Ando H, Ohno T, Asao T and Kuwano H: Clinical significance of melanoma antigen-encoding gene-1 (MAGE-1) expression and its correlation with poor prognosis in differentiated advanced gastric cancer. Ann Surg Oncol 18: 1195-1203, 2011.

45. Zhang S, Zhou X, Yu H and Yu Y: Expression of tumor-specific antigen MAGE, GAGE and BAGE in ovarian cancer tissues and cell lines. BMC Cancer 10: 163, 2010.

46. Zhou SJ, Zhuo SR, Yang XQ, Qin CX and Wang ZL: Serum Dickkopf-1 expression level positively correlates with a poor prognosis in breast cancer. Diagn Pathol 9: 161, 2014.

47. Shi Y, Gong HL, Zhou L, Tian J and Wang Y: Dickkopf-1 is a novel prognostic biomarker for laryngeal squamous cell carcinoma. Acta Otolaryngol 134: 753-759, 2014

48. Dong LL, Qu LY, Chu LY, Zhang XH and Liu YH: Serum level of DKK-1 and its prognostic potential in non-small cell lung cancer. Diagn Pathol 9: 52, 2014

49. Chen C, Zhou H, Zhang X, Ma X, Liu Z and Liu X: Elevated levels of Dickkopf-1 are associated with $\beta$-catenin accumulation and poor prognosis in patients with chondrosarcoma. PLoS One 9: e105414, 2014.

50. Huang Y, Yang X, Zhao F, Shen Q, Wang Z, Lv X, Hu B, Yu B, Fan J and Qin W: Overexpression of Dickkopf-1 predicts poor prognosis for patients with hepatocellular carcinoma after orthotopic liver transplantation by promoting cancer metastasis and recurrence. Med Oncol 31: 966, 2014.

51. Tao YM, Liu Z and Liu HL: Dickkopf-1 (DKK1) promotes invasion and metastasis of hepatocellular carcinoma. Dig Liver Dis 45: 251-257, 2013

52. Jiang T, Huang L and Zhang S: DKK-1 in serum as a clinical and prognostic factor in patients with cervical cancer. Int J Biol Markers 28: 221-225, 2013 